\title{
Strain variations in InGaAsP/InGaP superlattices studied by scanning probe microscopy
}

\author{
Huajie Chen and R. M. Feenstra ${ }^{a)}$ \\ Department of Physics, Carnegie Mellon University, Pittsburgh, Pennsylvania 15213 \\ R. S. Goldman ${ }^{\text {b) }}$ \\ Department of Materials Science and Engineering, The University of Michigan, Ann Arbor, Michigan 48109 \\ C. Silfvenius and G. Landgren \\ Department of Electronics, Royal Institute of Technology, Kista, Sweden
}

(Received 30 December 1997; accepted for publication 9 February 1998)

\begin{abstract}
Strain-compensated InGaAsP/InGaP superlattices are studied in cross section by atomic force microscopy and scanning tunneling microscopy. Undulations in the morphology of the $\{110\}$ cross-sectional faces are observed, and are attributed to elastic relaxation of this surface due to underlying strain arising from thickness and compositional variations of the superlattice layers. Finite element computations are used to extract a quantitative measure of the strain variation. (C) 1998 American Institute of Physics. [S0003-6951(98)03314-2]
\end{abstract}

The technique of cross-sectional scanning tunneling microscopy (XSTM) has been extensively used over the past five years to study III-V semiconductor heterostructures. ${ }^{1}$ These materials cleave easily along $\{110\}$ crystal faces, and those surfaces are not reconstructed and generally do not have surface states existing within the band gap of the bulk semiconductor. In that case, STM study reveals properties which are representative not only of the surface but also of the underlying material, thereby providing a wealth of information relating to, e.g., alloy fluctuations, interface roughness, band offsets, point defects, etc. in the semiconductor heterostructures. Despite this success, XSTM studies have remained relatively difficult to perform, requiring state of the art ultrahigh vacuum equipment and expertise in cleaving, probe tip preparation, and STM operation. In contrast, operation of an atomic force microscopy (AFM) is considerably easier, since it can be routinely used in ambient air conditions and allows scanning speeds (and zoom capabilities) which are typically ten times greater than for STM. Thus, it would be desirable to use the AFM for cross-sectional investigations, and indeed a number of such XAFM studies have been previously reported. ${ }^{2}$

In this work, we report on a new application of XAFM to the study of semiconductor heterostructures, in which the images are analyzed to reveal details of strain variations in the material. The strain compensated InGaAsP/InGaP/InP superlattices studied here have application for light sources and detectors in optical fiber communications systems, ${ }^{3}$ and strain variations are known to impact the optical efficiency of the devices. ${ }^{4,5}$ Strain inhomogeneities are revealed in these heterostructures by observing distortions of the nominally flat cross-sectional cleavage face due to the strain. This elastic relaxation gives rise to undulations of the surface, with magnitude of order $1 \mathrm{~nm}$ and lateral extent on the order of $100 \mathrm{~nm}$. Such undulations are easily measured with the AFM. We have used finite element modeling of the strain

\footnotetext{
${ }^{\text {a)} E l e c t r o n i c ~ m a i l: ~ f e e n s t r a @ a n d r e w . c m u . e d u ~}$

b)Electronic mail: rsgold@engin.umich.edu
}

relaxation to deduce the magnitude of the lateral strain inhomogeneities, and we relate this to variations in the thickness and alloy composition of the superlattice layers.

STM measurements are performed in an ultrahighvacuum chamber with base pressure $<5 \times 10^{-11}$ Torr. Images are obtained with a constant tunnel current of $0.1 \mathrm{nA}$, and at sample bias voltage specified below. The superlattice structures studied here consist of $5.0 \mathrm{~nm}$ thick $\mathrm{In}_{0.89} \mathrm{Ga}_{0.11} \mathrm{As}_{0.52} \mathrm{P}_{0.48}$ quantum wells, surrounded by barriers containing $\quad 4.9 \mathrm{~nm} \mathrm{In}_{0.89} \mathrm{Ga}_{0.11} \mathrm{P}+10.2 \mathrm{~nm} \mathrm{InP}+4.9 \mathrm{~nm}$ $\mathrm{In}_{0.89} \mathrm{Ga}_{0.11} \mathrm{P}$. The $\mathrm{In}_{0.89} \mathrm{Ga}_{0.11} \mathrm{As}_{0.52} \mathrm{P}_{0.48}$ and $\operatorname{In}_{0.89} \mathrm{Ga}_{0.11} \mathrm{P}$ have $0.9 \%$ compressive and $0.8 \%$ tensile lattice mismatch to the InP substrate, respectively. Details of the growth of these superlattices are provided elsewhere. ${ }^{5}$ AFM measurements are performed in ambient air conditions, using contact mode with a force of $6 \mathrm{nN}$. Sample cleavage is performed in ultrahigh vacuum (for STM) or in air (for AFM), with the (110) and (110) faces distinguished by anisotropic etching of the unpolished side of the (001) oriented wafers.

Figure 1(a) shows a constant-current STM image of the $\mathrm{InGaAsP} / \mathrm{InGaP} / \mathrm{InP}$ heterostructure. The individual quantum well and barrier layers are clearly visible. In addition, there is a larger scale modulation apparent in Fig. 1(a), with dark and light bands extending horizontally across the image. This topographic modulation is illustrated in the line cut of Fig. 1(b), which shows a $14 \AA$ peak-to-valley variation in the observed undulations. A similar type of undulation of the (110) cross-sectional face has also been observed for another heterostructure, grown without the $10 \mathrm{~nm}$ thick InP spacer layers in the barrier regions. ${ }^{5}$ Large variation in the thickness of the quantum wells and barriers was observed there, leading ultimately to severe structural degradation of the growth. In contrast, the superlattices pictured in Fig. 1 have relatively high structural quality, with relatively flat (001) interfaces between the layers, and uniform layer thicknesses. Nevertheless, a close examination of the data of Fig. 1 indicates that some small variations in the thickness of the layers are possible. Specifically, we find that the quantum wells and the barrier layers vary in thickness by approximately $\pm 2 \mathrm{~nm}$ 


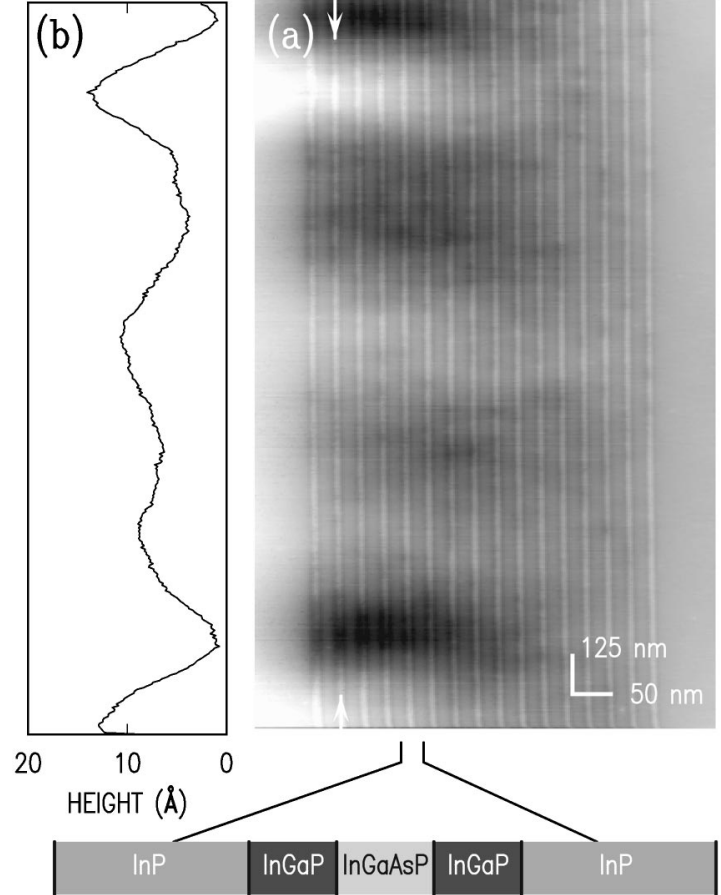

FIG. 1. (a) Cross-sectional STM image of the InGaAsP/InP/InGaP heterostructures, acquired at sample voltage of $2.5 \mathrm{~V}$. The vertical length scale in the image is compressed by a factor of 2.5 , as indicated. Tip height is indicated by a grey-scale, ranging from 0 (black) to $15 \AA$ (white). (b) Line cut through the STM image as the position indicated by arrows in (a). The observed undulations in the tip height are also seen as the alternating bright and dark bands in the image (a). A schematic view of the heterostructure is shown in the lower part of the figure.

over regions in the top half of the superlattice.

The undulations of the $(1 \overline{1} 0)$ face seen in Fig. 1 clearly indicate some sort of lateral inhomogeneity in the structures. Since the STM images are sensitive to both electronic and topographic effects, it is useful to separate these effects by performing an AFM study which measures only the true topographic variation. Figure 2 shows results of XAFM study of the heterostructures, including images obtained on both the $(1 \overline{1} 0)$ and (110) faces. The individual superlattice layers are faintly visible, extending vertically up each image. The observed corrugation amplitude of 1-2 $\AA$ between layers is consistent with that expected from elastic relaxation of the cleavage face due to the compressively and tensilely strained layers, ${ }^{6}$ as previously suggested by Pinnington et al. in a study of Si/Ge superlattice. ${ }^{7}$ More prominent are the distinct black and white regions in Fig. 2, producing an undulation of the cleavage surfaces extending laterally along the superlattice. The peak-to-valley amplitude of these undulation is about 16 and $14 \AA$ for Figs. 2(a) and 2(b), with a lateral period of 690 and 1200-2400 nm, respectively. Similar features have been imaged at other cleavage locations on the sample, and for other samples cut from the same wafer. The amplitude of the undulations varies (with the results of Fig. 2 representing maximum observed amplitudes), although their period is relatively constant for the $(1 \overline{10})$ cleavage faces.

Given that the undulation of the cleavage planes are seen not only by STM but also by AFM, they can be confidently assigned as purely topographic in origin. We attribute these features to elastic relaxation of the cleavage face, due to underlying regions which are under compressive or tensile
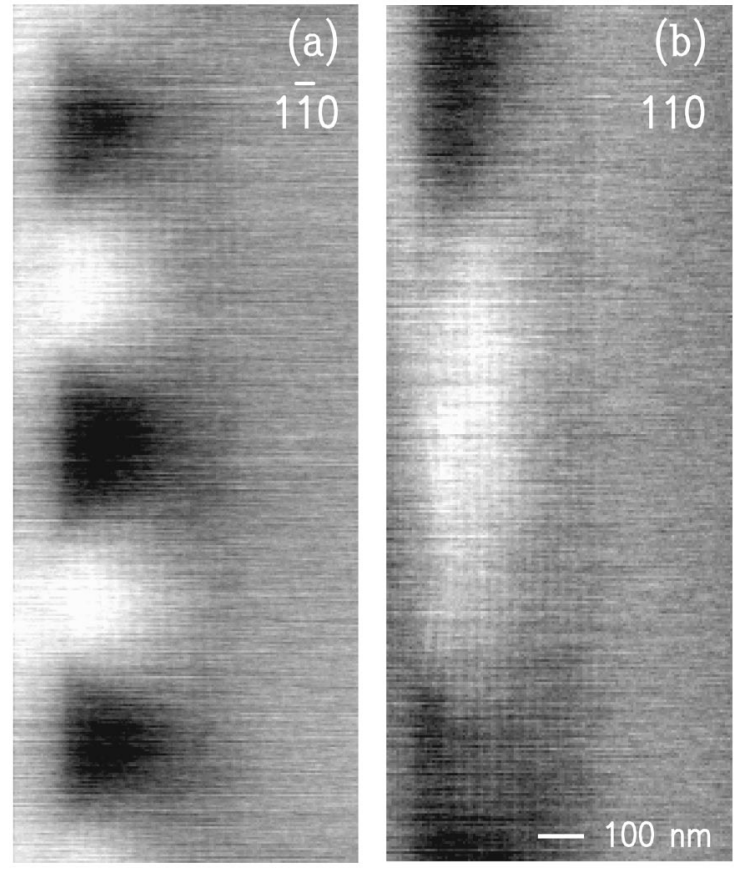

FIG. 2. Cross-sectional AFM images of the InGaAsP/InP/InGaP heterostructures, showing (a) (110) face and (b) (110) face. The superlattice periods are visible, and phase segregated regions appear as the protrusions (white) and depressions (black) on the surface. Grey-scale ranges are (a) 14 and (b) $12 \AA$.

stress. These stress variations are attributed to a combination of two effects: fluctuations in the thickness of the superlattice layers, and accompanying variation in the composition of the alloys in the layers. Similar effects have been reported in a number of semiconductor systems ${ }^{8-11}$ as studied by transmission electron microscopy (TEM), and are known to arise from surface stress-induced segregation of the alloy constituents during growth of the structures. The segregation effects and the morphological variations of the (001) growth surface are generally found to occur together; it is not currently known which (if any) of these effects acts as a driving force for the other.

The geometry of the segregated regions of the alloy is illustrated in the inset of Fig. 3. The superlattice (SL) region is found to be decomposed into a two-dimensional array of rectangular regions, with alternating strain of $\pm \epsilon$. In reality these strained regions will, of course, not form a well ordered array, but for computational convenience we assume this arrangement. The dimensions of the rectangular blocks, $a \times b$ $\times d$ are determined from experiment: from Fig. 2(a) we find $a=345 \mathrm{~nm}$ and from Fig. 2(b) we choose $d \approx 2 a=690 \mathrm{~nm}$. From the observed horizontal extent of the segregated regions in Fig. 2(a) we estimate $b \approx 2 a / 3=230 \mathrm{~nm}$. Given a particular geometrical arrangement, we can then compute the resulting elastic relaxation of the cleavage surface, using a finite elements technique. Computation are performed using ALGOR, using typically 13000 brick elements. The results are shown in Fig. 3, where we plot the peak-to-valley amplitude of the undulations $\Delta h$, normalized to $\epsilon a$.

Applying the general results shown in Fig. 3 to our specific geometry, we find a computed value of $\Delta h / \epsilon a$ of 1.57 . Using our maximum measured value of $\Delta h$ of $16 \AA$ (the observed undulation amplitude may be less than this, due to 


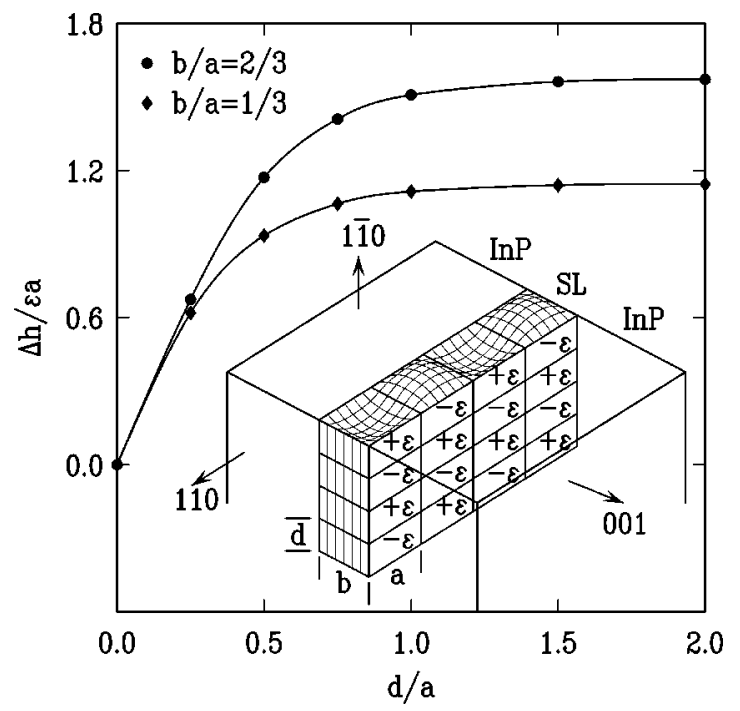

FIG. 3. Theoretical results from finite element computations for the magnitude of the surface undulations $\Delta h$, arising from a series of alternately strained regions at a surface. The inset shows the superlattice (SL) sandwiched between InP layers. Segregation of the SL alloy results in the array of rectangular regions with alternating strain $\pm \epsilon$. The dimensions of each rectangular region is $a \times b \times d$. Relaxation of the strain at the surface results in the undulation shown, with maximum amplitude $\Delta h$.

a cleavage plane which passes through some intermediate location near the center of the segregated regions), and with $a=345 \mathrm{~nm}$ from above, we find a relative strain between the phase segregated regions of $\epsilon= \pm 0.30 \%$. This strain can be allocated among the various superlattice layers in accordance with their known thicknesses and nominal compositions. For example, if we neglect the thickness variation and assume that all the strain arises from segregation of the cation species ( $\mathrm{Ga}$ and $\mathrm{In}$ ) we would predict a $\pm 7 \%$ variation in their composition (i.e., compositions of $\operatorname{In}_{0.96} \mathrm{Ga}_{0.04} \mathrm{P}$ and $\mathrm{In}_{0.82} \mathrm{Ga}_{0.18} \mathrm{P}$ in the ternary layers, and $\mathrm{In}_{0.96} \mathrm{Ga}_{0.04} \mathrm{As}_{0.52} \mathrm{P}_{0.48}$ and $\mathrm{In}_{0.82} \mathrm{Ga}_{0.18} \mathrm{As}_{0.52} \mathrm{P}_{0.48}$ and in the quaternary layers). If only the anion species (As and $\mathrm{P}$ ) in the relatively thin $\mathrm{In}_{0.89} \mathrm{Ga}_{0.11} \mathrm{As}_{0.52} \mathrm{P}_{0.48}$ layers segregate then we find a $\pm 46 \%$ variation in their concentration (which seems much too large to be likely). Alternatively, if we allow the barrier and well layers to vary in thickness by $\pm 2 \mathrm{~nm}$, with the sum of their thicknesses being a constant, then we find $\pm 4 \%$ compositional variations for purely cation segregation, or $\pm 25 \%$ variation for purely anion segregation. In general, we would expect that both cation and anions undergo some alloy segregation, yielding, e.g., $\pm 3 \%$ cation variation together with $\pm 6 \%$ anion variation. These values are comparable to the few $\%$ variations in alloy composition that have been observed in other systems. ${ }^{8-11}$

In conclusion, we have used cross-sectional STM and AFM to observe undulations on the $\{110\}$ cleavage faces of InGaAsP/InGaP/InP superlattices. These undulations are attributed to elastic relaxation of the surface due to underlying strain arising from thickness and alloy compositional variations. Notably, the phase segregation in this system proceeds in the absence of large modulation in the morphology of the (001) growth surface of the type seen in other systems. ${ }^{9,10}$ In those cases, the variation in alloy composition has been attributed to stress-induced variation in the growth rates of the alloy constituents (i.e., smaller atoms prefer small latticeconstant sites, and larger atoms prefer larger lattice-constant sites), with the source of the original stress variation being that arising from undulations in the growth surface of the stressed layer. $^{10,12}$ In our case, we believe that the same stress-induced segregation process applies, but the source of the stress is the phase segregation itself rather than some additional morphological variation in the growth surface. The process would begin with homogeneous nucleation of a group of atoms which are larger or smaller sized than the average, which in turn creates strain variations which favor the accumulation of similar-sized alloy species. ${ }^{11}$ This alloy segregation then continues and possibly is amplified as the growth proceeds. Morphological undulations of the growth surface would occur as a result of growth rates which vary with stress, but such undulations do not appear to act as the initial driving force for the stress-induced segregation process.

This work was supported by a grant from the National Science Foundation. Discussions with S. Mahajan, T. Tiedje, and D. G. Cahill are gratefully acknowledged. The authors thank I. K. Malcevic for assistance with the finite element computations.

${ }^{1}$ For reviews, see R. M. Feenstra, Semicond. Sci. Technol. 9, 2157 (1994); E. T. Yu, Mater. Sci. Eng., R. R17, 147 (1996).

${ }^{2}$ See, e.g., B. Dwir, F. Reinhardt, and E. Kapon, J. Appl. Phys. 78, 4939 (1995); G. Bratina, L. Vanzetti, and A. Franciosi, Phys. Rev. B 52, R8625 (1995); M. Barrett, M. Dennis, D. Tiffin, Y. Li, and C. K. Shih, J. Vac. Sci. Technol. B 14, 447 (1996); R. Alvis, B. Mantiply, and M. Young, ibid. 14, 452 (1996); A. J. Howard, O. Blum, H. Chui, A. G. Baca, and H. Crawford, Appl. Phys. Lett. 68, 3353 (1996).

${ }^{3}$ E. P. O'Reilly and A. R. Adams, IEEE J. Quantum Electron. 30, 366 (1994).

${ }^{4}$ C. Silfvenius, B. Stålnacke, and G. Landgren, J. Cryst. Growth 170, 122 (1997).

${ }^{5}$ R. S. Goldman, R. M. Feenstra, C. Silfvenius, B. Stålnacke, and G. Landgren, J. Vac. Sci. Technol. B 15, 1027 (1997).

${ }^{6}$ The elastic relaxation of the strained layers is computed using the same finite elements approach discussed in this letter. For the $\mathrm{InGaAsP} / \mathrm{InGaP} /$ InP heterostructure studied here, with strains of -0.91 and +0.78 in the $\mathrm{In}_{0.89} \mathrm{Ga}_{0.11} \mathrm{As}_{0.52} \mathrm{P}_{0.48}$ and $\mathrm{In}_{0.89} \mathrm{Ga}_{0.11} \mathrm{P}$ layers respectively, we find that the quaternary layers protrude out from the surface and the ternary layers contract into the surface, resulting in a peak-to-valley corrugation amplitude of $1.0 \AA$

${ }^{7}$ T. Pinnington, A. Sanderson, T. Tiedje, T. P. Pearsall, E. Kasper, and H. Presting, Thin Solid Films 222, 259 (1992).

${ }^{8}$ K. Y. Cheng, K. C. Hsieh, and J. N. Baillargeon, Appl. Phys. Lett. 60, 2892 (1992); P. J. Pearah, A. C. Chen, A. M. Moy, K. C. Hsieh, and K. Y. Cheng, IEEE J. Quantum Electron. 30, 608 (1994).

${ }^{9}$ A. Ponchet, A. Rocher, A. Ougazzaden, and A. Mircea, J. Appl. Phys. 75, 7881 (1994).

${ }^{10}$ G. C. Hua, N. Otsuka, D. C. Grillo, J. Han, L. He, and R. L. Gunshor, J. Cryst. Growth 138, 367 (1994).

${ }^{11}$ G. Patriarche, A. Ougazzaden, and F. Glas, Appl. Phys. Lett. 69, 2279 (1996).

${ }^{12}$ K. Lee, W. C. Johnson, and S. Mahajan, Inst. Phys. Conf. Ser. 146, 235 (1995). 